\title{
BubR1 is required for the mitotic block induced by combretastatin-A4 and a novel cis-restricted B-lactam analogue in human cancer cells
}

\author{
LISA M. GREENE ${ }^{1}$, MIRIAM CARR $^{2}$, NIALL O. KEELEY ${ }^{2}$, MARK LAWLER $^{3}$, \\ MARY J. MEEGAN ${ }^{2}$ and DANIELA M. ZISTERER ${ }^{1}$ \\ Schools of ${ }^{1}$ Biochemistry and Immunology and ${ }^{2}$ Pharmacy, Trinity College Dublin; \\ ${ }^{3}$ Institute of Molecular Medicine, St. James's Hospital and Trinity College Dublin, Dublin 2, Ireland
}

Received September 3, 2010; Accepted November 4, 2010

DOI: $10.3892 / \mathrm{ijmm} .2011 .633$

\begin{abstract}
BubR1 is a well-defined guardian of the mitotic spindle, initiating mitotic arrest in response to the lack of tension and/or chromosome alignment across the mitotic plate. However, the role of BubR1 in combretastatin-induced cell death remains unknown. In this study, we describe the effects of combretastatin A-4 (CA-4) and a synthetic cisrestricted 3,4-diaryl-2-azetidinone (ß-lactam) analogue (CA-432) on the modulation and phosphorylation of BubR1 in human cervical cancer-derived cells. We demonstrate that CA-4 and CA-432 depolymerise the microtubular network of human cervical carcinoma-derived cells. Both compounds induced the disassembly of the microtubules and the loss of microtubule tension led to the early phosphorylation of BubR1 and the late cleavage of BubR1. The phosphorylation of BubR1 correlated with the onset of $\mathrm{G}_{2} \mathrm{M}$ cell cycle arrest whilst the cleavage of BubR1 coincided with apoptosis induced by the combretastatins. The combretastatin-induced apoptosis and the BubR1 cleavage were caspase-dependent. In vitro enzyme digests demonstrated that combretastatinactivated BubR1 is a substrate for caspase-3. Gene silencing of BubR1 with small interfering RNA severely compromised combretastatin-induced $\mathrm{G}_{2} \mathrm{M}$ cell cycle arrest with a corresponding increase in the formation of polyploid cells in both cervical and breast cancer-derived cells. In summary, BubR1 is required to maintain the $\mathrm{G}_{2} \mathrm{M}$ arrest and limit the formation of polyploid cells in response to continued combretastatin exposure. Moreover, substitution of the ethylene bridge with 3,4-diaryl-2-azetidinone did not alter the tubulin depolymerising properties or the subsequent mitotic spindle checkpoint response to CA-4 in human cancer cells.
\end{abstract}

Correspondence to: Dr Lisa Greene, School of Biochemistry and Immunology, Trinity College Dublin, College Green, Dublin 2, Ireland

E-mail: greeneli@tcd.ie

Key words: combretastatin-A4, tubulin, BubR1, cis-restricted analogues

\section{Introduction}

The cis-stilbene combretastatin-A4 (CA-4) was originally isolated from the African willow tree, Combretum caffrum, and remains one of the most potent anti-cancer agents of natural origin (1). CA-4 is structurally and functionally similar to the microtubule targeting agent (MTA), colchicine. MTAs function by directly interacting with tubulin and either promoting (tubulin polymerisers) or inhibiting (tubulin depolymerisers) the assembly of tubulin dimers, thereby altering the natural equilibrium between free tubulin dimers and assembled polymers (2). CA-4 inhibits the assembly of tubulin by interacting with tubulin at or near the colchicine binding site (3). Due to solubility issues, disodium combretastatin A-4 3-0-phosphate (CA-4P) was subsequently synthesised as a water soluble pro-drug of CA-4 (4). CA-4P is rapidly metabolised by endogenous phosphatases into the parent compound. CA4-P was the first MTA to inhibit tumour blood flow at one-tenth the maximum tolerated dose (5). For this reason, CA4-P entered clinical trials as a vascular targeting agent (VTA). VTAs range from MTAs to cytokines and were designed to cause rapid and extensive shutdown of the established tumour vasculature (6). Phase I clinical trials demonstrated rapid changes in tumour vasculature in response to CA-4P, with Phase II clinical trials demonstrating significant clinical activity in patients with anaplastic thyroid cancer (7-10). Given the profound success of CA-4P in combination with other chemotherapeutic agents in animal models, CA-4P in combination therapy is currently undergoing eagerly awaited clinical evaluation in patients with advanced cancer (11).

It is well documented that both the anti-vascular, anticancer and tubulin-targeting properties of combretastatin are highly dependent on the cis-configuration of the double bond between the two aryl rings and the 3,4,5-trimethoxy systems on the A ring (12). Cis-trans isomerisation readily occurs in heat, light and protic media and thus limits the potential therapeutic index of this class of VTAs. In order to combat this downfall, many lines of research have focused on a number of strategies to overcome double bond isomerisation, most of which involve the insertion of heterocyclic rings in place of the usual ethylene bridge. We recently designed and 
synthesised a novel series of cis-restricted CA-4 analogues, substituting the ethylene bridge structure for a 3,4-diaryl-2azetidinone ( $\beta$-lactam) ring (13). The rigid $\beta$-lactam ring scaffold allows a similar spatial arrangement between the two phenyl rings as observed in the cis-conformation of CA-4, while permanently preventing the undesired conversion to the inactive trans-configuration. Addition of various moieties to the B-lactam ring lead to the development of more potent analogues, including CA-432 [chemical name, 4-(3-hydroxy4-methoxyphenyl)-3-phenyl-1-(3,4,5-trimethoxyphenyl)azetidin-2-one]. CA-432 is structurally similar to the previously described CA-12d [chemical name, 4-(3-hydroxy3-methoxyphenyl)-1-(3,4,5-trimethoxyphenyl)azetidin-2one] (13) with the exception of an additional phenyl ring at position 3 on the $\beta$-lactam ring structure. The addition of the phenol ring increased the potency of the $\beta$-lactam series of CA-4 analogues by up to 5-fold (14).

In addition to its anti-vascular properties, CA-4 is also a potent anti-cancer agent due to its high affinity for tubulin and subsequent destabilisation of mitotic spindles in rapidly proliferating cancer cells. CA-4 and related compounds are hence classified as MTAs. Accordingly, CA-4P depolymerised the microtubules and induced the mitotic arrest and subsequent cell death of mitotic endothelial cells following prolonged drug exposure (15). Furthermore, CA-4 and many other synthetic derivatives depolymerised the microtubular network and induced cell death in various cancer cells by both caspase-dependent and -independent mitotic catastrophe cell death $(16,17)$. CA-4P-induced mitotic arrest was associated with increased cyclin-B protein levels in the chronic lymphocytic leukemia cell line, WSU-CLL (17). No changes in the expression of many other key regulators of $\mathrm{G}_{2} \mathrm{M}$ cell cycle arrest, including MDM2, 14-3-3sigma, GADD45, the cyclindependent kinase cdc2, its inhibitory phosphorylation, the cdc2-inhibitory kinase (wee1), chk1, or cdc25 hyperphosphorylation, were associated with $\mathrm{CA}-4 \mathrm{P}$-induced mitotic arrest in human CLL cells (17). A recent study demonstrated an association of increased survivin (a member of the inhibitor of apoptosis protein family) expression with resistance to CA-4-related compounds in human oral carcinoma cells (18). The down-regulation of survivin by small interfering RNA (siRNA) increased the sensitivity of CA-4-related compounds in these cells. Despite significant advances over the years, the exact mechanism of CA-4-induced cell death is not yet fully understood.

Given that MTAs perturb the mitotic spindles and trigger mitotic arrest during cell division, significant interest in the involvement of members of the spindle assembly checkpoint (SAC) in MTA-induced cell death has escalated over the years. The SAC monitors both the attachment of chromosomes to the mitotic spindle and the tension across the sister chromatids generated by microtubules. MTAs can interfere with microtubule dynamics during mitosis by stabilising or destabilising the microtubules, thereby altering the tension across the mitotic spindle leading to the activation of the SAC. Many key components of the SAC have been identified, including Bub1, BubR1, Mps1, Bub3 and Mad1-3, which interact with $\mathrm{Cdc}-20$ and prevent cell cycle progression to anaphase by inhibiting the activity of the anaphase-promoting complex/cyclosome (APC/C) (19). The APC/C is an ubiquitin ligase which, following interaction with its co-activator, Cdc-20, targets anaphase inhibitors and mitotic cyclins for degradation by the proteasome (20). BubR1, also known as Bub1-related kinase or MAD3/Bub1b, is a principle component of the SAC and plays an essential role in the recruitment of other spindle checkpoint proteins to the kinetochore during mitosis (21). BubR1 together with BUB3, Cdc-20 and MAD2, form the mitotic checkpoint complex (MCC) which prevents premature mitotic exit by the inhibition of the APC/C complex (22). The MCC APC/C inhibitory activity is 3,000-fold greater than recombinant MAD2 alone (21). The phosphorylation of BubR1 is essential for monitoring the stable spindle attachments to kinetochores and the tension and for an active SAC (23). Kinases such as Cdk1, Aurora B and PIK1, have been implicated in the phosphorylation and thus the activation of BubR1 (24-26). Activated BubR1 binds to and thus inhibits the activity of Cdc-20, the co-activator of the APC/C and thereby prevents progression to anaphase (25). This pathway is essential for the induction of a mitotic arrest in response to the tubulin polymeriser, paclitaxel (27). The degradation of BubR1 by both a caspase-dependent and a ubiquitindependent proteosome pathway, have been associated with mitotic release after prolonged mitotic spindle damage $(28,29)$. BubR1 was recently identified as a substrate of the APC/C (30). The acetylation status of BubR1 was identified as the critical determinant in the molecular switch of BubR1 from an inhibitor to a substrate of the APC/C (30).

Given the pivotal role of BubR1 in both normal mitotic progression and cellular response to mitotic insult, we sought to determine the effect of CA-4 exposure on the modulation of BubR1 in human cancer cells. We also sought to determine whether ethylene bridge substitutions of CA-4 influence the biochemical signalling pathway of the parent compound.

\section{Materials and methods}

Compounds. The CA-4 and B-lactam analogue, CA-432. was synthesised as previously described (14). Both drugs were dissolved in ethanol at $10 \mathrm{mM}$ solutions and stored in the dark at $-20^{\circ} \mathrm{C}$. Z-VAD general caspase inhibitor (Merck Chemicals Ltd., Nottingham, UK) was dissolved in DMSO and used at a final concentration of $50 \mu \mathrm{M}$. The final concentrations of solvents were $0.2 \%(\mathrm{v} / \mathrm{v})$ for ethanol and $0.1 \%(\mathrm{v} / \mathrm{v})$ for DMSO. CA-4 was synthesised according to the protocols described by Carr et al (13). CA-432 is novel analogue of CA-4. It is structurally similar to CA-12d, a synthetic derivative of CA-4 with the exception of an additional phenyl ring at $\mathrm{C} 3$ on the $\beta$-lactam ring (13) (Fig. 1). The synthesis of CA-432 was described previously (14).

Cells. Cell lines were originally obtained from the European Collection of Cell Cultures (Salisbury, UK). HeLa cells are cervical adenocarcinoma-derived and MDA-MB-231 cells originate from a pleural effusion from a patient with metastatic adenocarcinoma of the breast. Cultures were grown in Dulbecco's Modified Eagle's Medium (DMEM) with glutamax supplemented with $10 \%$ FBS, 100 units/ml penicillin and $100 \mu \mathrm{g} / \mathrm{ml}$ streptomycin (Invitrogen, CA). Cells were maintained at $37^{\circ} \mathrm{C}$ in $5 \% \mathrm{CO}_{2}$ in a humidified incubator. 
Combretastatin -A4 (CA-4)

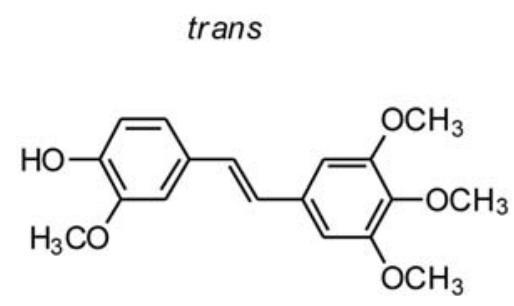

cis<smiles>COc1ccc(/C=C\c2cc(OC)c(OC)c(OC)c2)cc1O</smiles>

CA-12d
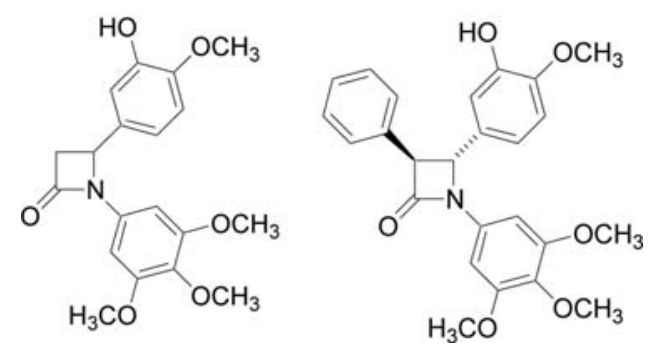

Figure 1. Structures of CA-4 in both the trans (inactive) and cis (active) conformation and B-lactam analogues, CA-12d and CA-432.

Immunofluorescence and confocal microscopy. For immunofluorescence, HeLa cells were grown on poly-L-lysine-coated coverslips. Following treatment, the cells were washed gently in PBS and fixed for $30 \mathrm{~min}$ in methanol at $-20^{\circ} \mathrm{C}$. Following washes in PBS and $0.1 \%$ Triton X-100 (PBST), cells were blocked in 5\% BSA diluted in PBST (blocking buffer). Cells were then incubated with mouse anti-tubulin (DM1A) (Merck Chemicals Ltd.), 1:20 for $1 \mathrm{~h}$ at room temperature. Following washes in PBST, the cells were incubated with secondary antibodies (Alexa 488 anti-mouse) for $1 \mathrm{~h}$ at room temperature. Following washes in PBST, the cells were stained with propidium iodide (PI) at $0.2 \mu \mathrm{g} / \mathrm{ml}$ in PBS for 2 min and mounted in 4\% propyl gallate in PBS:glycerol. Confocal images were captured using the Olympus 1 X81 microscope coupled with Olympus Fluoview version 1.5 software. All images in each experiment were collected on the same day using identical parameters.

Tubulin polymerisation assay. Tubulin depolymerisation was quantified using a modified version of a method originally documented by Minotti et al (31). Briefly, following treatment, the cells were pelleted, washed in PBS and harvested into microtubule-preserving buffer [0.1M Pipes ( $\mathrm{pH}$ 6.9), $2 \mathrm{M}$ glycerol, $5 \mathrm{mM} \mathrm{MgCl}_{2}, 2 \mathrm{mM}$ EGTA, $0.5 \%$ Triton X-100, and EDTA protease inhibitor cocktail tablets (Roche)] supplemented with $4 \mu \mathrm{M}$ taxol. The taxol is required to maintain the stability of assembled microtubules during the isolation procedure. The soluble fraction (containing depolymerised tubulin) was separated from the insoluble fraction (containing polymerised tubulin) by centrifugation at 16,000 $\mathrm{x} g$ for $45 \mathrm{mins}$ at $4^{\circ} \mathrm{C}$. The insoluble fraction was then re-

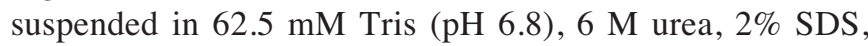
$10 \%$ glycerol and $0.00125 \%$ bromophenol blue, and briefly sonicated. Equal amounts of each sample were analysed by Western blotting using anti-tubulin mAb (DM1A; 1/1000 dilution).

Determination of DNA content. Following treatment, the cells were fixed with $70 \%$ ethanol overnight at $-20^{\circ} \mathrm{C}$. Following fixation, FBS $[0.25 \%(\mathrm{v} / \mathrm{v})]$ was added to the cells prior to centrifugation. The cell pellets were re-suspended in PBS containing $10 \mu \mathrm{g} / \mathrm{ml}$ RNase A and $100 \mu \mathrm{g} / \mathrm{ml}$ PI and incubated at $37^{\circ} \mathrm{C}$ for $30 \mathrm{~min}$. The PI fluorescence was measured on a linear scale using a FACScalibur flow cytometer (Becton-Dickinson, San Jose, CA). Data collection was gated to exclude cell debris and cell aggregates. At least 10,000 cells were analysed per sample. All the data were recorded and analysed using the CellQuest software (BectonDickinson).

Western blot analysis. The BubR1 and Poly (ADP-ribose) polymerase (PARP) protein levels were determined by Western blot analysis as previously described (32). Mouse anti-human BubR1 was purchased from BD Transduction Laboratories (Cowley, UK). Anti-BubR1 detects both the phosphorylated and unphosphorylated form of BubR1. Mouse anti-actin mAb and anti-PARP were supplied from Merck Chemicals Ltd.

siRNA transfection. In this study, we used a validated siRNA duplex to target the BubR1 sequence 5'-GGUGGGAAGG AGAGUAAUATT-3' (Ambion, UK). A scrambled RNA duplex was used as the control (5'-AGGGUAGUAGGAGAU GATT-3'). The selected siRNAs were BLAST searched against the human genome sequence to ensure that only one gene was targeted, whereas the control scrambled siRNA sequence used, had no known overlap. Cells were seeded at a density of $7.2 \times 10^{3} / \mathrm{cm}^{2}$ (HeLa) and $1.8 \times 10^{4} / \mathrm{cm}^{2}$ (MDA-MB231 ) in DMEM without antibiotics. After $24 \mathrm{~h}$, transfection was carried out on $50 \%$ confluent cells in Optimen medium using Oligofectamine Transfection Reagent (Invitrogen) in accordance with the manufacturer's instructions. The final concentration for the siRNAs was $50 \mathrm{nM}$. The siRNA complexes were removed after $4 \mathrm{~h}$ and replaced with DMEM supplemented with $20 \%$ FCS. The cells were treated $24 \mathrm{~h}$ post-transfection with the vehicle $[0.2 \%$ ethanol (v/v)], CA-4 $(50 \mathrm{nM})$ or CA-432 (50 nM) for $48 \mathrm{~h}$.

In vitro BubRl cleavage. We then sought to determine whether BubR1 or activated (phosphorylated) BubR1 are susceptible to caspase- 3 cleavage. HeLa cells were exposed to the vehicle $[0.2 \%$ ethanol $(\mathrm{v} / \mathrm{v})], \mathrm{CA}-4(50 \mathrm{nM})$, or CA-432 $(50 \mathrm{nM})$ for $18 \mathrm{~h}$. BubR1 was phosphorylated following an 18-h exposure to CA-4 or CA-432. Following treatment, 
A

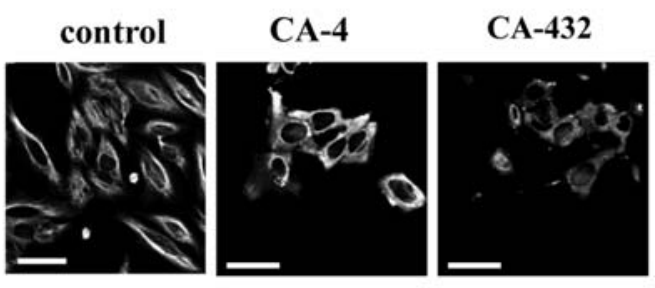

B

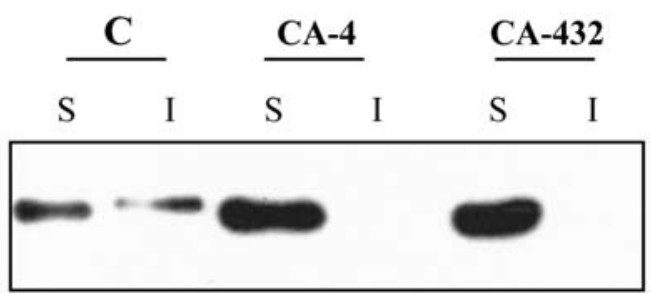

Figure 2. CA-4 and CA-432 depolymerise the microtubular network of human cervical cells. HeLa cells were exposed to the vehicle $[0.2 \%$ ethanol $(\mathrm{v} / \mathrm{v})]$, CA-4 (50 nM) or CA-432 (50 nM) for $24 \mathrm{~h}$. (A) Confocal images of cells stained with DM1A anti- $\alpha$-tubulin mAb (white). Scale bars represent $50 \mu \mathrm{M}$. (B) Following treatment with anti-mitotic agent, cytosolic (S, soluble unpolymerised tubulin) and cytoskeleton (I, insoluble polymerised tubulin) tubulin fractions were separated by centrifugation and analysed by Western blotting using anti- $\alpha$-tubulin mAb. Results are representative of three independent experiments.

HeLa cells $\left(4 \times 10^{6}\right)$ were lysed in $100 \mu l$ of caspase cleavage buffer (20 mM HEPES-NaOH (pH 7.5), 10\% (w/v) sucrose, $0.1 \%(\mathrm{w} / \mathrm{v})$ CHAPS, $1 \mathrm{mM}$ EDTA and protease inhibitors. Protein extracts $(50 \mu \mathrm{g})$ were incubated with/or without 120 units of caspase- 3 for $3 \mathrm{~h}$ at $37^{\circ} \mathrm{C}$. Reactions were terminated by the addition of SDS sample buffer and boiling. Digests were then separated by SDS-PAGE, blotted to PVDF and probed with anti-BubR1 and anti-actin antibodies.

Statistical analysis. The statistical analysis of experimental data was performed using the Student's paired t-test and the results are presented as the mean \pm SEM. A value of $\mathrm{P}<0.05$ was considered to be significant.

\section{Results}

CA-4 and a novel $\beta$-lactam derivative, CA-432, inhibit the polymerisation of tubulin, induce cell cycle arrest and delay apoptosis in HeLa cells. CA-432 is structurally (in terms of orientation) (Fig. 1) and biologically similar (in terms of effects on tubulin polymerisation and cell viability $\mathrm{IC}_{50}$ values) to CA-4 (14). Hence, for this study, both drugs were used at a concentration of $50 \mathrm{nM}$. The exposure of cells to MTAs predominantly results in the disorganisation of the microtubular network, malformed mitotic spindles, mitotic cell cycle arrest and in most instances, apoptosis. Hence, we first determined the effects of CA-4 and CA-432 on the microtubular network and the cell cycle. Confocal analysis of HeLa cells stained with $\alpha$-tubulin mAb demonstrated a dense microtubular network in the control cells (Fig. 2A). Exposure
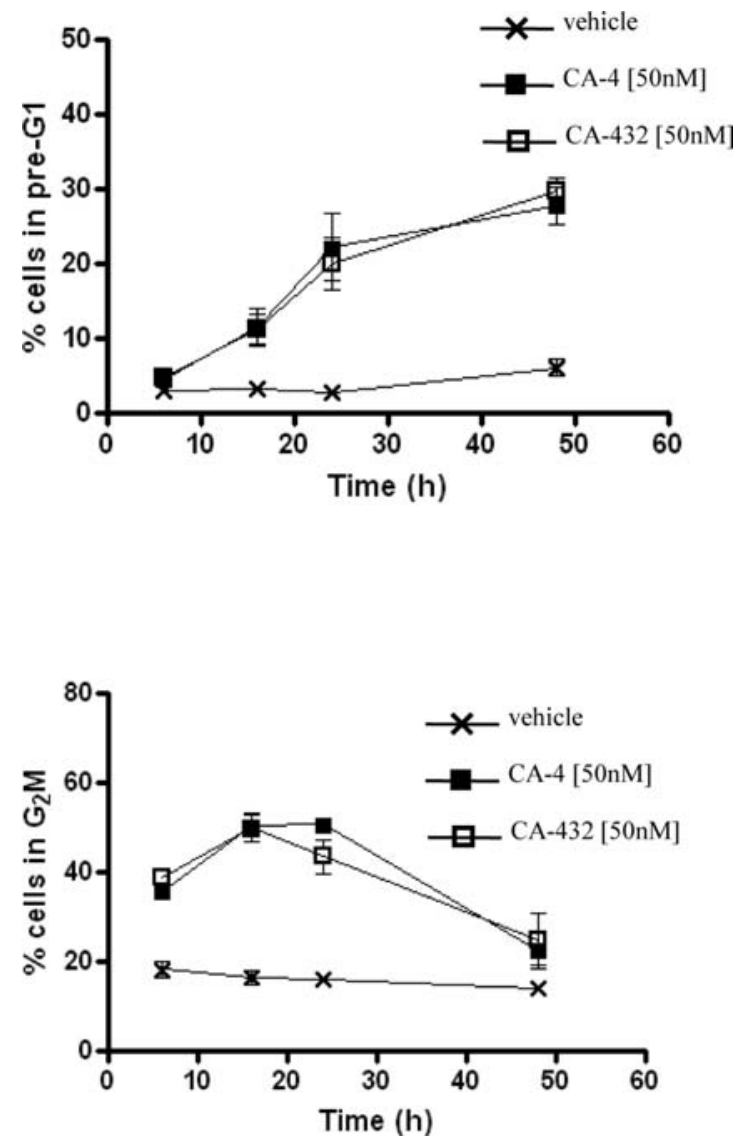

Figure 3. CA-4 and CA-432 induce $\mathrm{G}_{2} \mathrm{M}$ cell cycle arrest and apoptosis in HeLa cells. HeLa cells were exposed to the vehicle [0.2\% ethanol (v/v)], CA-4 $(50 \mathrm{nM})$ or CA-432 $(50 \mathrm{nM})$ for the times indicated. Cells were then fixed, stained with PI and analysed by flow cytometry. Cell cycle analysis was performed on histograms of gated counts per DNA area (FL2-A). The number of cells with $<2$ (sub-G1), $2\left(\mathrm{G}_{0} \mathrm{G}_{1}\right)$ and $4 \mathrm{~N}\left(\mathrm{G}_{2} \mathrm{M}\right)$ DNA content was determined using the CellQuest software. The sub-G1 peak is indicative of apoptosis. Values represent the means \pm SEM of at least three separate experiments. The absence of error bars indicates that the error was smaller than the size of the symbol.

to the microtubule depolymeriser, $\mathrm{CA}-4$, and the novel $\mathrm{B}$ lactam derivative, CA-432, for $24 \mathrm{~h}$ led to the disorganisation of the microtublar network with diffuse tubulin staining (Fig. $2 \mathrm{~A}$; center and right panel, respectively). Sedimentation assays followed by Western blotting demonstrated an equal distribution of tubulin in soluble and insoluble fractions in the control cells. In contrast, the distribution of tubulin in the CA-4- and CA-432-treated cells shifted to the soluble extract containing depolymerised tubulin (Fig. 2B). Flow cytometric analysis of CA-4 and the B-lactam analogue CA-432-treated cells demonstrated a time-dependent increase in the accumulation of cells in the $\mathrm{G}_{2} \mathrm{M}$ phase of the cell cycle followed by a decline at $24 \mathrm{~h}$ as the number of apoptotic cells increased (Fig. 3). Collectively, these results demonstrate that both CA-4 and its novel analogue, CA-432, targeted the microtubules and induced mitotic cell arrest followed by delayed apoptosis in HeLa cells.

Cleavage of the mitotic spindle checkpoint regulator, BubR1, is associated with the onset of apoptosis induced by CA-4 and the CA-432 $\beta$-lactam analogue. To date, there is limited 


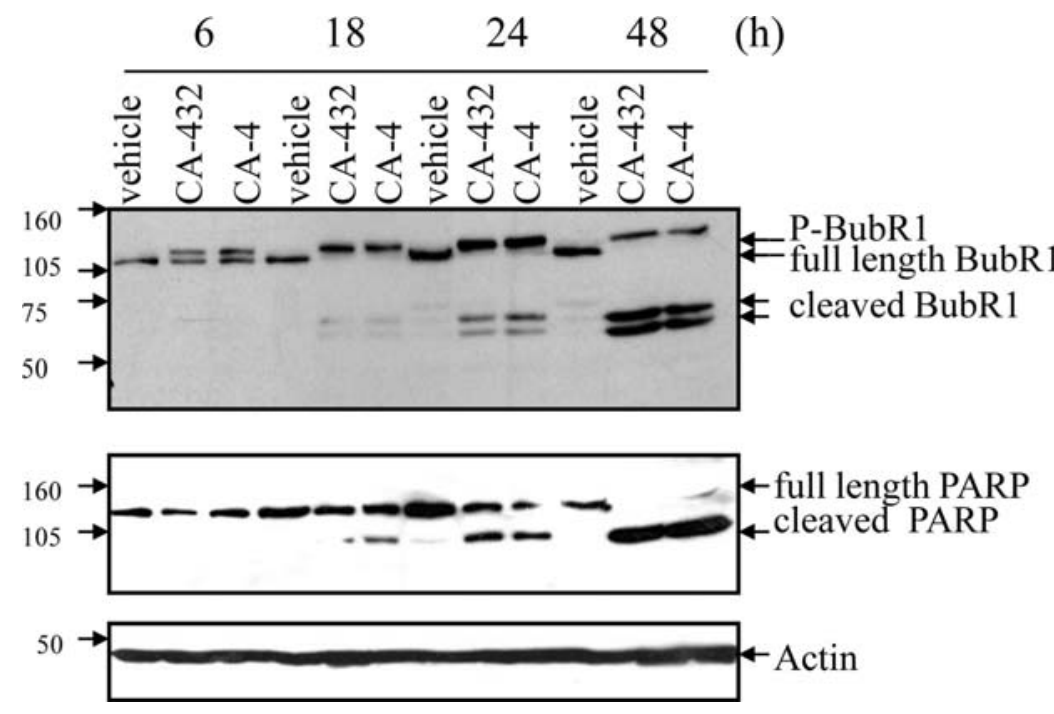

Figure 4. BubR1 cleavage is associated with CA-4 and CA-432-induced apoptosis. HeLa cells were exposed to the vehicle [0.2\% ethanol (v/v)] CA-4 (50 $\mathrm{nM})$ or CA-432 (50 nM) for the times indicated. Whole cell lysates were resolved by SDS-PAGE and probed with anti-BubR1 mouse mAb. The anti-BubR1 mAb detects both the phosphorylated and unphosphorylated form of BubR1. P-BubR1 indicates the phosphorylated BubR1 protein. Blots were probed with antiactin $\mathrm{mAb}$ as the loading control. Results are representative of three separate experiments.

knowledge regarding the biochemical components of the combretastatin apoptotic cascade. BubR1 has been identified as a critical component in the mitotic cell cycle block induced by other classes of MTAs and DNA targeting agents. The phosphorylated form of BubR1 has been consistently linked with an active spindle checkpoint (23). Hence, we examined the effect of CA-4 and its analogue on the phosphorylation of BubR1 during mitotic arrest and apoptosis. The antibody used in this experiment was raised against a.a 276-388 and detects both the phosphorylated and unphosphorylated form of BubR1 (32). The phosphorylation of BubR1 is detected by the presence of a slower migrating band by SDS-PAGE. The Western blotting results demonstrate that both CA-4 and CA-432-induced the phosphorylation of BubR1 (Fig. 4). Two lower molecular weight fragments were also detected in the CA-4 and CA-432 protein extracts migrating at $\sim 60$ and $70 \mathrm{kDa}$. These smaller fragments most likely represent the cleaved forms of BubR 1 generated by the proteolysis of BubR1. The appearance of these cleaved fragments coincided with the onset of apoptosis induced by both compounds in HeLa cells. Apoptosis was determined by quantification of the sub-G1 peak and PARP cleavage. PARP is cleaved into two fragments (89- and 24-kDa) during apoptosis. Such cleavage renders the enzyme inactive by destroying its ability to respond to DNA strand breaks. These findings suggest that the phosphorylation of BubR1 could facilitate a mitotic block following mitotic insult by the combretastatins. Moreover, the cleavage of BubR1 could allow mitotic exit.

Gene silencing of BubR1 does not sensitise human cancer cells to combretastatin-induced apoptosis. We then sought to investigate whether BubR1 protein levels are important for combretastatin-induced mitotic block and apoptosis. siRNA repression of BubR1 was confirmed by Western blot analysis (Fig. 5A). Cells were transfected with scrambled or BubR1 siRNA $(50 \mathrm{nM})$ complexes for $4 \mathrm{~h}$. At $24 \mathrm{~h}$ post-transfection, cells were then treated with CA-4 or CA-432 for $48 \mathrm{~h}$. The effects of BubR1 repression of the cell cycle profiles of HeLa cells exposed to CA-4 and CA-432, were assessed by flow cytometry. Knockdown of BubR1 induced spontaneous apoptosis in HeLa cells (Fig. 5B). This lethality is due to massive chromosome loss (17). The treatment of BubR1depleted HeLa cells with CA-4 or CA-432 did not significantly enhance the level of spontaneous apoptosis induced by BubR1 knockdown. BubR1 knockdown was not lethal in MDA-MB-231 cells. A significant reduction in CA-432induced cell death was observed in MDA-MB-231 cells following BubR1 repression (Fig. 5D). These data suggest that the loss of a functional spindle checkpoint increases resistance to CA-432. It is also possible that the apoptosis observed in BubR1-depleted HeLa cells exposed to CA-4 and CA-432 is due to BubR1 knockdown alone, as opposed to microtubule insult. Prolonged exposure to the combretastatins led to a significant increase $(\mathrm{P}<0.01)$ in cellular polyploidy in BubR1-depleted cancer cells with a corresponding decrease in the percentage of cells blocked in $\mathrm{G}_{2} \mathrm{M}$ (Fig. 5C). Cells became polyploid ( $>4 \mathrm{~N}$ DNA) due to repeated endoreplication without cell division. A significant increase $(\mathrm{P}<0.02)$ in polyploidy with a corresponding decrease in the percentage of cells in $G_{2} M$ and those undergoing apoptosis was also observed in a second cell line, MDA-MB-231, in response to CA-432 (Fig. 5D and E). Collectively, the results high-lighted here demonstrate a role for BubR1 in maintaining a stringent mitotic block in response to continued exposure to combretastatins and thereby limiting the formation of polyploid cells.

Combretastatin-induced apoptosis and BubRl cleavage are caspase-dependent. CA-4 induces caspase-dependent as well as -independent cell death in cells of different neoplastic origin $(16,17)$. Hence, we sought to determine whether caspases are involved in CA-4-induced cell death in cervical cancer cells using the general caspase inhibitor, Z-VAD- 

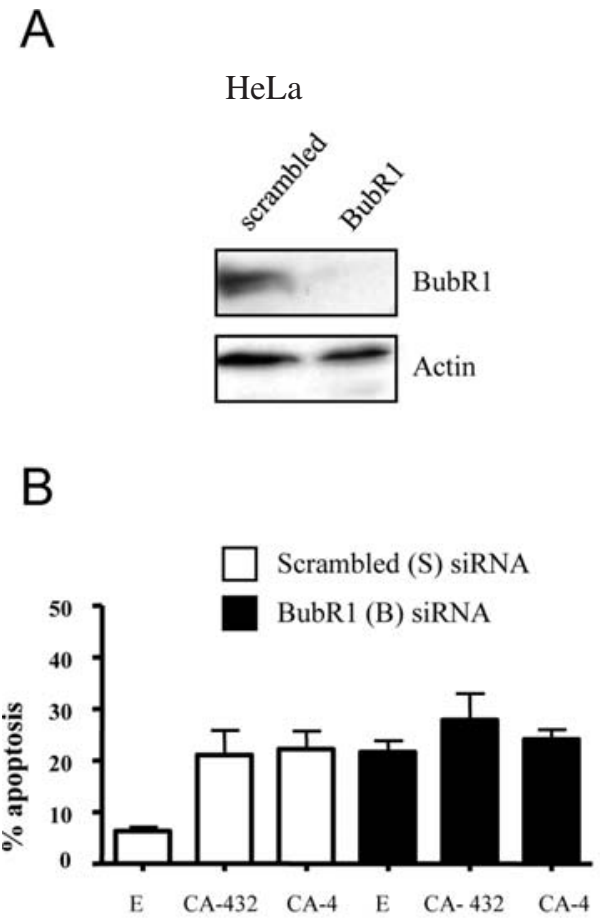

D

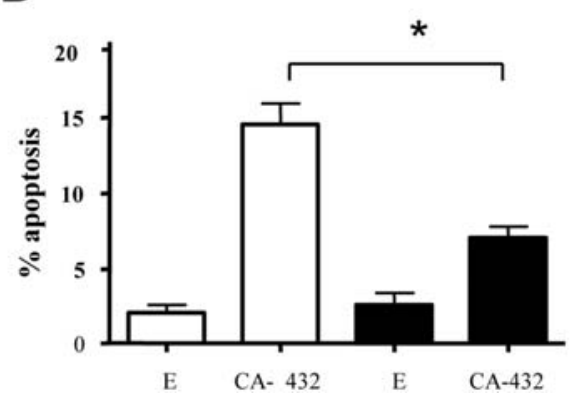

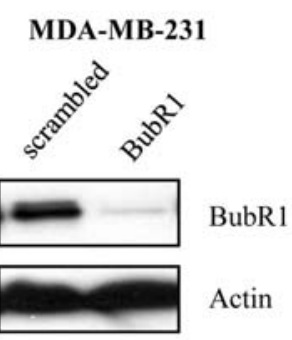

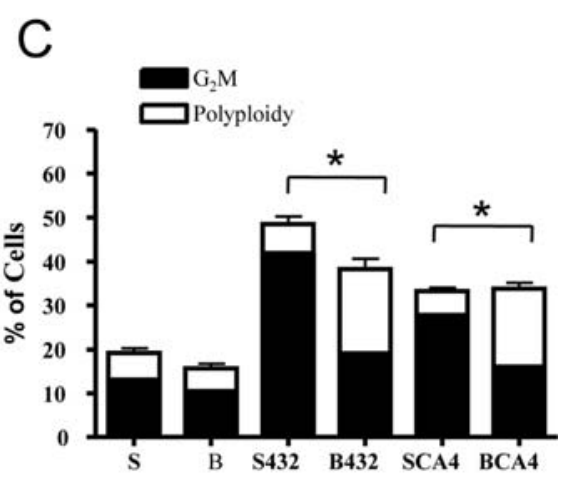

E

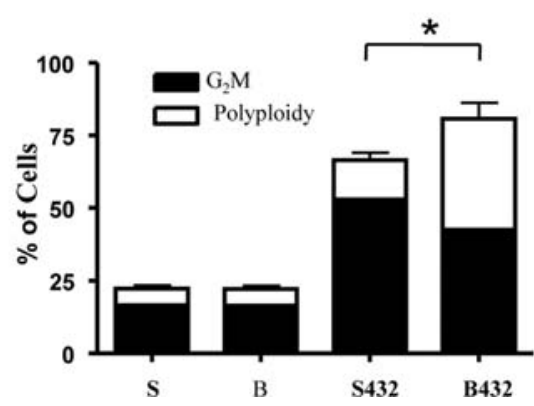

Figure 5. siRNA repression of BubR1 promotes polyploidy but not apoptosis following mitotic insult by CA-4 and CA-432. HeLa and MDA-MB-231 cells were exposed to siRNA duplexes for $4 \mathrm{~h}$ after which the medium was replaced with DMEM medium containing $20 \%$ FBS for $20 \mathrm{~h}$. (A) Untreated whole cell lysates were harvested $48 \mathrm{~h}$ post-transfection with scrambled or BubR1 siRNA complexes. The final concentration of siRNA was $50 \mathrm{nM}$. Equal amounts of protein were separated by SDS-PAGE and probed with anti-BubR1 mAb. Blots were probed with anti-actin mAb as the loading control. (B-E) After $24 \mathrm{~h}$ post-transfection with siRNA duplexes, cells were then exposed to the ethanol vehicle (E) [0.2\% (v/v)], or CA-4 (50 nM) or CA-432 (50 nM) for $48 \mathrm{~h}$. Cell cycle analysis was performed on the histograms of gated counts per DNA area (FL2-A). Relative percentage of cells with $<2$ (sub-G1; apoptotic), 2 ( $\mathrm{G}_{0} \mathrm{G}_{1}$ ) and $4\left(\mathrm{G}_{2} \mathrm{M}\right)$ or $>4 \mathrm{~N}$ (polyploid) DNA content were derived from the analysis of DNA histograms using the CellQuest software. Results are representative of at least three separate experiments. Values represent the means \pm SEM of three to five separate experiments. ( ${ }^{*} \mathrm{P}<0.05$; paired Student's t-test).

FMK. As shown in Fig. 6, the pre-treatment of HeLa cells with Z-VAD-FMK completely abrogated CA-4- and CA-432induced apoptosis (Fig. 6A) and BubR1 cleavage (Fig. 6B). Given that PARP is cleaved by caspase-3 during apoptosis, PARP cleavage was assessed as a hallmark of apoptosis and as a marker for active caspases. As we had anticipated, PARP cleavage coincided with the CA-4- and CA-432induced apoptosis and accordingly PARP cleavage was inhibited by Z-VAD-ZMK (Fig. 6B). The failure of the combretastatin-treated cells to undergo apoptosis following the inhibition of caspases was associated with a sustained mitotic block (Fig. 6A). Collectively, these findings suggest that the caspase cleavage of BubR1 is essential for mitotic release and subsequent apoptosis in response to the mitotic insult by CA-4.
In vitro cleavage of BubR1 by caspase-3. The complete inhibition of the combretastatin-induced BubR1 cleavage by the general caspase inhibitor, Z-VAD-FMK suggests that BubR1 could be a caspase substrate. Using the CASVM programme (www.casbase.org), 45 potential caspase cleavage sites, including 5 predicted caspase- 3 cleavage sites, were identified within the BubR1 amino acid sequence. Given that BubR1 is cleaved at two sites by caspase- 3 during paclitaxel-induced cell death (28) coupled with the presence of 5 D-X-X-D motifs, we determined the susceptibility of BubR1 to caspase- 3 cleavage using in vitro digests. Extracts from the control untreated and combretastatin-treated cells containing inactive (unphosphorylated) and activated (phosphorylated) BubR1, respectively, were exposed to caspase-3. Western blot analysis of caspase- 3 digests demonstrated that 
A
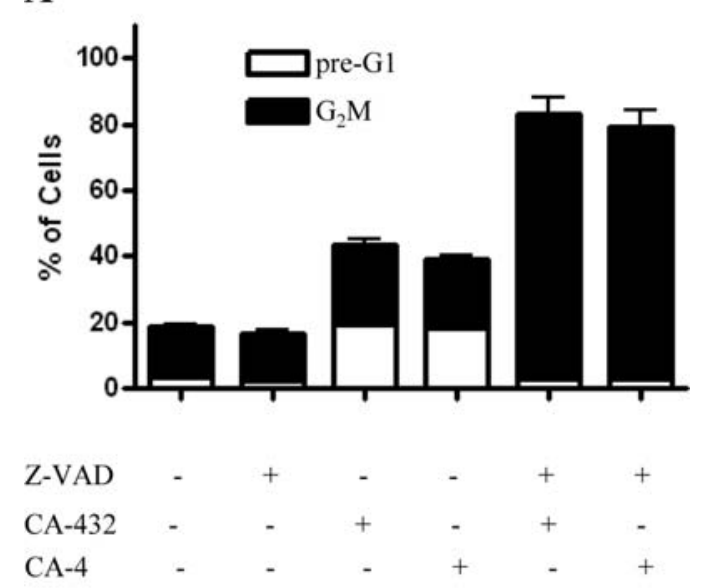

B

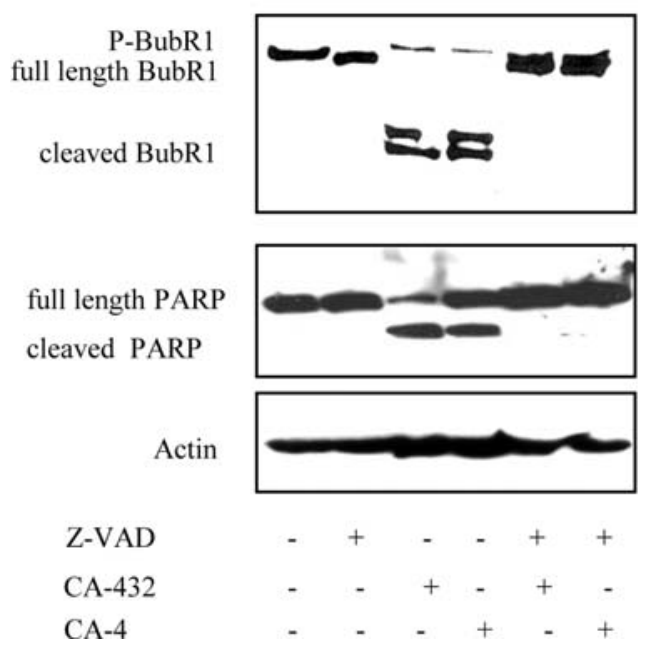

Figure 6. The general caspase inhibitor, z-VAD, inhibits CA-4 and CA-432induced apoptosis, as well as BubR1 and PARP cleavage. Subconfluent HeLa cells were pre-treated with the general caspase inhibitor, Z-VAD $(50 \mu \mathrm{M})$, for $1 \mathrm{~h}$ followed by exposure to the ethanol vehicle $[0.2 \%(\mathrm{v} / \mathrm{v})] \mathrm{CA}-4(50 \mathrm{nM})$ or CA-432 (50 nM) for $48 \mathrm{~h}$. (A) Cell cycle profiles were determined by flow cytometry. The percentage of apoptosis was assessed by quantification of the sub-G1 peak. (B) Whole cell lysates were resolved by SDS-PAGE and probed with anti-BubR1, anti-PARP and anti-actin mouse mAbs Results are representative of three separate experiments.

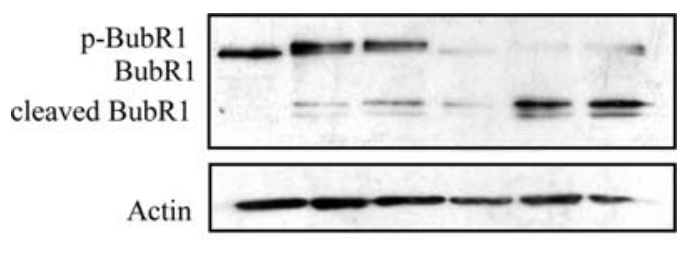

$\begin{array}{lllllll}\text { Lane } & 1 & 2 & 3 & 4 & 5 & 6 \\ \text { CA-432 } & - & + & - & - & + & - \\ \text { CA-4 } & - & - & + & - & - & + \\ \text { C3 } & - & - & - & + & + & +\end{array}$

Figure 7. In vitro digestion of BubR1 with purified recombinant caspase-3. HeLa cells were exposed to the vehicle [ethanol $0.2 \%$ (v/v)], CA-4 (50 nM) or CA-432 (50 nM) for $18 \mathrm{~h}$. Lysates were incubated with/without caspase-3 (C3) for $3 \mathrm{~h}$ at $37^{\circ} \mathrm{C}$. Digests were then separated by SDS-PAGE, blotted to PVDF and probed with anti-BubR1 and anti-actin antibodies. Intact and major breakdown products are indicated.
BubR1 is cleaved by caspase-3 (Fig. 7). The levels of endogenous inactive BubR1 were reduced by caspase-3, indicating that BubR1 is indeed a substrate of caspase- 3 . (Fig. 7, lane 4). Cleavage products with a similar migration pattern to that observed during combretastatin-induced apoptosis were more prominent in extracts containing the active BubR1 (Fig. 7, lanes 5 and 6). This finding shows that phosphorylated BubR1 is potentially cleaved by caspase- 3 during combretastatin-induced apoptosis.

\section{Discussion}

There is no disputing the fact that BubR1 is an essential gene. Gene knockout of BubR1 in mice leads to early embryonic death due to massive apoptosis (33). Colony outgrowth assays of siRNA BubR1-depleted HeLa cells demonstrated complete cell death after 6 divisions without BubR1 (34). Furthermore, the deletion of just a single BubR1 allele in mice severely compromised the mitotic checkpoint function, yielding higher levels of chromosome missegregation and an increased susceptibility to carcinogen-induced carcinomas (35). Apart from normal mitotic cell function, BubR1 is also essential for mitotic checkpoint response following exposure to the tubulin polymeriser, paclitaxel, the tubulin depolymerisers, colcemid and arsenic trioxide, and a novel series of tubulin depolymerisers, the Pyrrolo[1,5]benzoxa(thia)zepines $(26,32,34,36)$. Also, a recent study demonstrated a role for BubR1 in $\mathrm{G}_{2} \mathrm{M}$ cell cycle arrest in response to DNA damage induced by the topoisomerase inhibitor, doxorubicin (37). The phosphorylation of BubR1 is essential for monitoring the stable spindle attachments to kinetochores and the tension and for an active SAC (22). It has been shown that the absolute levels of BubR1 determine the length of mitosis (38). Increased levels of BubR1 promote a more stringent mitotic response to MTAs (38). siRNA experiments targeting BubR1 decreased the length of mitosis and resulted in an impaired mitotic response to MTAs $(27,39)$. Additionally, a decrease in BubR1 expression was noted in cells containing aligned chromosomes in late metaphase with levels further declining with progression to anaphase (40). Furthermore, the caspase-mediated cleavage of BubR1 is essential for mitotic exit in response to exposure to the tubulin depolymeriser, nocodazole (28).

In this study, we highlight the essential role of BubR1 during mitotic arrest and apoptosis in response to the VTAs, the combretastatins. We also demonstrate that the phosphorylation and thus the activation of BubR1 is correlated with the mitotic arrest induced by the combretastatins in HeLa cells. In a separate study, we also observed the phosphorylation and activation of BubR1 following combretastatin exposure in leukemia cells (data not shown). In this study, the cleavage of BubR1 correlated temporally with the onset of apoptosis induced by CA-4 and its B-lactam analogue, CA-432. Consistent with this finding, the pan-specific caspase inhibitor Z-VAD-FMK completely abrogated combretastatin-induced apoptosis and BubR1 cleavage. Furthermore, in vitro digests of HeLa lysates containing active (phosphorylated) BubR1 were susceptible to caspase-3 cleavage. It can therefore be suggested that the cleavage of BubR1 by caspase- 3 can release BubR1 from the MCC, 
thereby attenuating the mitotic spindle checkpoint and consequently facilitating the onset of apoptosis. siRNA studies targeting BubR1 support the hypothesis that this mitotic spindle checkpoint protein is essential for a stringent mitotic block in response to combretastatin-induced microtubule insult. In support of this hypothesis, the genetic depletion of BubR1 in a second cell line, MDA-MB-231, compromised mitotic checkpoint in response to CA-432. An increase in cellular polyploidy was observed in BubR1depleted cells following prolonged exposure (48 h) to CA-4 and CA-432. These findings compliment the observations made by Shin et al (29), in which a decrease in BubR1 protein levels was associated with polyploid formation in nocodazole-treated cells. Collectively, these findings undoubtedly support the opinion that BubR1 is required for an effective mitotic checkpoint in response to CA-4- and CA4-related compounds, with a decrease in BubR1 protein levels required for mitotic release. A significant decline in the percentage of apoptotic cells was observed in BubR1depleted cells exposed to the synthetic combretastatin, CA432 in MDA-MB-231 breast cancer cells. An independent study by Sudo et al (27) also demonstrated the dependence of paclitaxel sensitivity in breast cancer cells on a functional spindle checkpoint. Furthermore, substitution of the ethylene bridge with a $\beta$-lactam ring did not influence the biological properties of the parent compound, CA-4.

\section{Acknowledgements}

Sincere thanks to Dr Orla Hanrahan (School of Biochemistry and Immunology, Trinity College Dublin) for her technical assistance on the confocal microscope. We would like to thank the Health Research Board of Ireland and the Higher Education Authority of Ireland for funding our study.

\section{References}

1. Pettit GR, Cragg GM and Singh SB: Antineoplastic agents, 122 Constituents of Combretum caffrum. J Nat Prod 50: 386-391, 1987.

2. Pasquier E and Kavallaris M: Microtubules: a dynamic target in cancer therapy. IUBMB Life 60: 165-170, 2008.

3. McGown AT and Fox BW: Structural and biochemical comparison of the anti-mitotic agents colchicine, combretastatin A4 and amphethinile. Anticancer Drug Des 4: 249-254, 1989.

4. Chaplin DJ and Hill SA: The development of combretastatin A4 phosphate as a vascular targeting agent. Int J Radiat Oncol Biol Phys 54: 1491-1496, 2002.

5. Dark GG, Hill SA, Prise VE, Tozer GM, Pettit GR and Chaplin DJ: Combretastatin A-4, an agent that displays potent and selective toxicity toward tumor vasculature. Cancer Res 57: 1829-1834, 1997.

6. Thorpe PE: Vascular targeting agents as cancer therapeutics. Clin Cancer Res 10: 415-427, 2004.

7. West CM and Price P: Combretastatin A4 phosphate. Anticancer Drugs 15: 179-187, 2004.

8. Petit I, Karajannis MA, Vincent L, Young L, Butler J, Hooper AT, Shido K, Steller H, Chaplin DJ, Feldman E and Rafii S: The microtubule-targeting agent CA4P regresses leukemic xenografts by disrupting interaction with vascular cells and mitochondrialdependent cell death. Blood 111: 1951-1961, 2008.

9. Anderson HL, Yap JT, Miller MP, Robbins A, Jones T and Price PM: Assessment of pharmacodynamic vascular response in a phase I trial of combretastatin A4 phosphate. J Clin Oncol 21: 2823-2830, 2003.

10. Cooney MM, Ortiz J, Bukowski RM and Remick SC: Novel vascular targeting/disrupting agents: combretastatin A4 phosphate and related compounds. Curr Oncol Rep 7: 90-95, 2005 .
11. Rustin GJ, Shreeves G, Nathan PD, Gaya A, Ganesan TS, Wang D, Boxall J, Poupard L, Chaplin DJ, Stratford MR, Balkissoon $J$ and Zweifel M: A Phase Ib trial of CA4P (combretastatin A-4 phosphate), carboplatin, and paclitaxel in patients with advanced cancer. Br J Cancer 102: 1355-1360, 2010.

12. Pettit GR, Rhodes MR, Herald DL, Hamel E, Schmidt JM and Pettit RK: Antineoplastic agents. 445. Synthesis and evaluation of structural modifications of (Z)- and (E)-combretastatin A-41. J Med Chem 48: 4087-4099, 2005.

13 Carr M, Greene LM, Knox AJ, Lloyd DG, Zisterer DM and Meegan MJ: Lead identification of conformationally restricted B-lactam type combretastatin analogues: synthesis, antiproliferative activity and tubulin effects. Eur J Med Chem: Sept. 22, 2010 (Epub ahead of print).

14. O'Boyle N, Carr M, Greene L, Bergin O, Nathwani S, McCabe T, Lloyd D, Zisterer D, Meegan M: Synthesis and evaluation of azetidinone analogues of combretastatin A-4 as tubulin targetin agent. J Med Chem (In press)

15. Kanthou C, Greco O, Stratford A, Cook I, Knight R, Benzakour O and Tozer G: The tubulin-binding agent combretastatin A-4phosphate arrests endothelial cells in mitosis and induces mitotic cell death. Am J Pathol 165: 1401-1411, 2004

16. Vitale I, Antoccia A, Cenciarelli C, Crateri P, Meschini S, Arancia G, Pisano C and Tanzarella C: Combretastatin CA-4 and combretastatin derivative induce mitotic catastrophe dependent on spindle checkpoint and caspase-3 activation in non-small cell lung cancer cells. Apoptosis 12: 155-166, 2007.

17. Nabha SM, Mohammad RM, Dandashi MH, Coupaye-Gerard B, Aboukameel A, Pettit GR and Al-Katib AM: CombretastatinA4 prodrug induces mitotic catastrophe in chronic lymphocytic leukemia cell line independent of caspase activation and poly(ADP-ribose) polymerase cleavage. Clin Cancer Res 8: 2735-2741, 2002

18. Cheung $\mathrm{CH}$, Chen $\mathrm{HH}$, Kuo CC, Chang CY, Coumar MS, Hsieh HP and Chang JY: Survivin counteracts the therapeutic effect of microtubule de-stabilizers by stabilizing tubulin polymers. Mol Cancer 8: 43, 2009.

19. Sczaniecka MM and Hardwick KG: The spindle checkpoint: how do cells delay anaphase onset? SEB Exp Biol Ser 59: 243-256, 2008

20. Peters JM: The anaphase-promoting complex: proteolysis in mitosis and beyond. Mol Cell 9: 931-943, 2002.

21. Chen RH: BubR1 is essential for kinetochore localization of other spindle checkpoint proteins and its phosphorylation requires Mad1. J Cell Biol 158: 487-496, 2002.

22. Sudakin V, Chan GK and Yen TJ: Checkpoint inhibition of the $\mathrm{APC} / \mathrm{C}$ in HeLa cells is mediated by a complex of BUBR1, BUB3, CDC20, and MAD2. J Cell Biol 154: 925-936, 2001.

23. Huang H, Hittle J, Zappacosta F, Annan RS, Hershko A and Yen TJ: Phosphorylation sites in BubR1 that regulate kinetochore attachment, tension, and mitotic exit. J Cell Biol 183: 667-680, 2008.

24. Wong OK and Fang G: Cdk1 phosphorylation of BubR1 controls spindle checkpoint arrest and Plk1-mediated formation of the 3F3/2 epitope. J Cell Biol 179: 611-617, 2007.

25. Ditchfield C, Johnson VL, Tighe A, Ellston R, Haworth C, Johnson T, Mortlock A, Keen N and Taylor SS: Aurora B couples chromosome alignment with anaphase by targeting BubR1, Mad2, and Cenp-E to kinetochores. J Cell Biol 161: 267-280, 2003.

26. Elowe S, Hummer S, Uldschmid A, Li X and Nigg EA: Tension-sensitive Plk1 phosphorylation on BubR1 regulates the stability of kinetochore microtubule interactions. Genes Dev 21: 2205-2219, 2007

27. Sudo T, Nitta M, Saya H and Ueno NT: Dependence of paclitaxel sensitivity on a functional spindle assembly checkpoint. Cancer Res 64: 2502-2508, 2004

28. Kim M, Murphy K, Liu F, Parker SE, Dowling ML, Baff W and Kao GD: Caspase-mediated specific cleavage of BubR1 is a determinant of mitotic progression. Mol Cell Biol 25: 9232-9248, 2005.

29. Shin HJ, Baek KH, Jeon AH, Park MT, Lee SJ, Kang CM, Lee HS, Yoo SH, Chung DH, Sung YC, McKeon F and Lee CW: Dual roles of human BubR1, a mitotic checkpoint kinase, in the monitoring of chromosomal instability. Cancer Cell 4: 483-497, 2003.

30. Choi E, Choe H, Min J, Choi JY, Kim J and Lee H: BubR1 acetylation at prometaphase is required for modulating APC/C activity and timing of mitosis. EMBO J 28: 2077-2089, 2009. 
31. Minotti AM, Barlow SB and Cabral F: Resistance to antimitotic drugs in Chinese hamster ovary cells correlates with changes in the level of polymerized tubulin. J Biol Chem 266: 3987-3994, 1991.

32. Greene LM, Campiani G, Lawler M, Williams DC and Zisterer DM: BubR1 is required for a sustained mitotic spindle checkpoint arrest in human cancer cells treated with tubulintargeting pyrrolo-1,5-benzoxazepines. Mol Pharmacol 73: 419-430, 2008

33. Wang Q, Liu T, Fang Y, Xie S, Huang X, Mahmood R, Ramaswamy G, Sakamoto KM, Darzynkiewicz Z, Xu M and Dai W: BUBR1 deficiency results in abnormal megakaryopoiesis. Blood 103: 1278-1285, 2004.

34. Kops GJ, Foltz DR and Cleveland DW: Lethality to human cancer cells through massive chromosome loss by inhibition of the mitotic checkpoint. Proc Natl Acad Sci USA 101: 8699-8704, 2004.

35. Dai W, Wang Q, Liu T, Swamy M, Fang Y, Xie S, Mahmood R, Yang YM, Xu M and Rao CV: Slippage of mitotic arrest and enhanced tumor development in mice with BubR1 haploinsufficiency. Cancer Res 64: 440-445, 2004.
36. Wu WC, Yen WY and Yih LH: Requirement of a functional spindle checkpoint for arsenite-induced apoptosis. J Cell Biochem 105: 678-687, 2008.

37. Fang Y, Liu T, Wang X, Yang YM, Deng H, Kunicki J, Traganos F, Darzynkiewicz Z, Lu L and Dai W: BubR1 is involved in regulation of DNA damage responses. Oncogene 25: 3598-3605, 2006

38. Chan GK, Jablonski SA, Sudakin V, Hittle JC and Yen TJ: Human BUBR1 is a mitotic checkpoint kinase that monitors CENP-E functions at kinetochores and binds the cyclosome/ APC. J Cell Biol 146: 941-954, 1999.

39. Meraldi P, Draviam VM and Sorger PK: Timing an checkpoints in the regulation of mitotic progression. Dev Cell 7: 45-60, 2004.

40. Howell BJ, Moree B, Farrar EM, Stewart S, Fang G and Salmon ED: Spindle checkpoint protein dynamics at kinetochores in living cells. Curr Biol 14: 953-964, 2004. 